\title{
Full-waveform inversion: Filling the gaps
}

\author{
Wafik B. Beydoun ${ }^{1}$ and Tariq Alkhalifah ${ }^{2}$
}

After receiving an outstanding response to its inaugural workshop in 2013, SEG once again achieved great success with its 2015 SEG Middle East Workshop, "Full-waveform inversion: Filling the gaps," which took place 30 March-1 April 2015 in Abu Dhabi, UAE. The workshop was organized by SEG, and its partner sponsors were Saudi Aramco (gold sponsor), ExxonMobil, and CGG.

With more than 70 participants, the workshop highlighted the challenges, difficulties, and solutions - impending or already proved - in full-waveform inversion (FWI) to test their applications in resolving the onshore near surface, shallow water, unconventional resources, and offshore subsalt. Eight sessions with two dedicated poster discussions and approximately 33 paper presentations were spaced across the three-day workshop. Participants were given approximately 45 minutes for brainstorming and discussion at the end of each session.

With its high-caliber speakers, the workshop served as a platform to share and discuss the strengths and weaknesses of novel FWI techniques. The workshop also provided a forum for discussion of the wide spectrum of FWI involvement, i.e., research and development; economic exploration and development; enhanced oil recovery; and acquisition, processing, interpretation, and so forth. Workshop participants came from distinct geographic regions, including the Middle East, Europe, United States, and Asia, and from institutions, including Saudi Aramco, ADNOC, Kuwait Oil Company, ADCO, Mubadala Petroleum, ADMA, Shell, Schlumberger, Total, ExxonMobil, CGG, INOVA, CNRS Geosciences Azur, JOGMEG, KAUST, the Petroleum Institute, ETH Zurich, Seoul National University, Wave Research, and PGS.

The workshop's introductory session focused on the methods of acquiring data for FWI. Among the topics discussed in the session were whether supergathers compromise spatial resolution, the possible requirement of supergrouping techniques if low frequencies deem reliable, and the cost-effectiveness of vibrators and very long sweeps for low frequencies.

The second session featured discussions about acoustic FWI and its role in the seismic-processing workflow. Questions arose as to how the gap between tomography and FWI could be dissolved, with probable solutions being FWI-guided tomography or high resolution from the former and background from the latter. Suggestions to update the near-surface land FWI with Laplace and/or Fourier solutions will diminish the disturbance caused in the traditional objective function. The MAZ marine FWI case study was the final topic of discussion before the close of the second session.

Speakers in the third session discussed the importance of frequency as a solution for multiparameter inversion. To further prove their theory, they demonstrated applications of FWI to elastic, viscoacoustic, and anisotropic media. A new dimension to the elastic inversion of land data - known to eradicate ground roll in field data, further preventing the generation of surface waves - was highlighted at this session. Speakers also stressed the need to include all the relevant physical phenomena to produce more substantial modeled data. Among the many ideas discussed, the importance of accounting for multiples at lower frequencies was emphasized.

For the reformulation of FWI, discussed in the fourth session, full-model wavenumber inversion (FMWI), which classifies the background and image/perturbation individually, was suggested. Diving waves are modeled in the background only, reflected energy using Born modeling with the background and image, but these modeled data are then combined into a single term in the objective function. The resulting scattering-angle domain filters control the respective updates.

The ideology behind FWI was on the same lines as Tariq Alkhalifah's approach, with slight discrepancy on model separation, which is achieved by impedance-velocity parameterization - the impedance providing the reflectivity — with them using separate terms in the objective function for both the diving and reflected waves. Through the medium of video recording, Art Weglein compared FWI with his direct inversion, which is based on a geometric series representation giving way to a closed-form solution or other useful algebraic manipulations.

The session concluded with poster presentations by the speakers. KAUST garnered maximum attention with its presentation, which displayed a multicomponent inversion in acoustic TI media, an evolution of Dix-type inversion for anisotropic media, FWI methodology using estimated scattering angles, and analysis of parameter resolution for divingwave tomography. Other presentations included an algorithm for local time-stepping for efficient wave simulation in heterogeneous media, allowing for errors in the physics of the forward equations in FWI, and workflows using parameterizations adapted to the differential operators in the modeling equations.

An insight into seismic simulation techniques was provided in the fifth session, "Modeling HPC." Another crucial aspect that surfaced in the meeting was how inversion engine physics would abide by the concept of simulation. At the end of the session, speakers suggested a particular strategy for efficient wavefield compression which, according to them, was to "judge the quality of the compression by the gradient, not the forward modeling."

Performances and constraints were analyzed in the workshop's sixth session. A low-cost approach was discussed for adaptation to further assess the resolution using random perturbations. Incorporating nonseismic information consistently 
and devising innovative approaches to predict HPC were the other factors suggested for better performance and eliminating any constraints.

Reflection tomography needs to be complemented by a surface-wave inversion and diving-wave inversion and divingwave plus refraction-tomography technique for near-surface and land data. Alternatively, horizontally adjusting topography creates tremendous effects on surface waves, and any assumptions similar to the $1 \mathrm{D}$ model are considered rather inconsistent in this case. Abnormalities observed in Middle East data seem similar to the head waves scattered by topographic. During this session on near-surface/land data, the last topic of deliberation was the effect of smooth parameterization on the convergence of FWI at larger frequencies.

Reservoir characterization dominated the event's final session, in which the resolution limit was pushed one notch ahead. It was also observed that FWI forms a natural tool to predict perturbations or changes from legacy data, in which such changes are represented in the model rather than the data, which is a desired goal of our industry.

At the end of the eight sessions, it was concluded that FWI and its utility have certain impending challenges and require

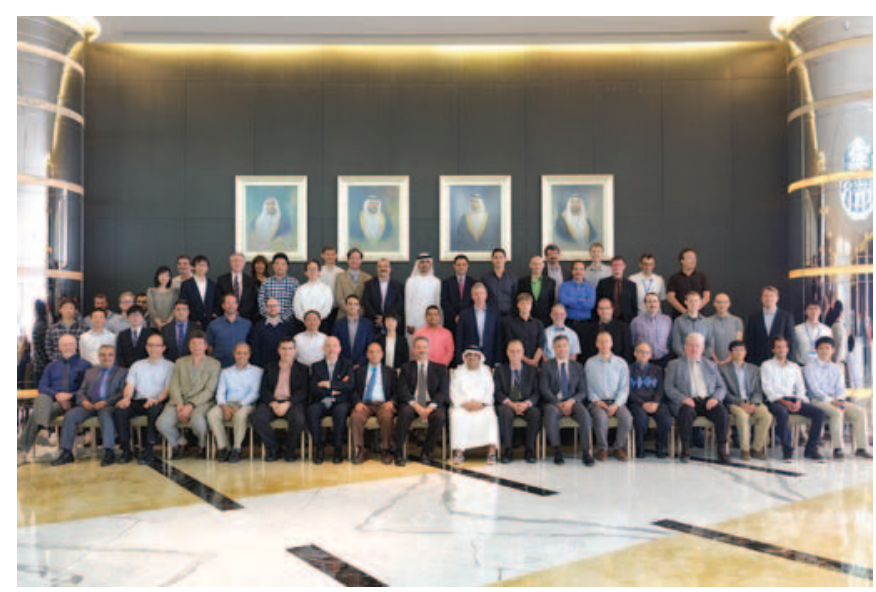

The FWI Workshop hosted more than 70 participants from the Middle East, Europe, the United States, and Asia.

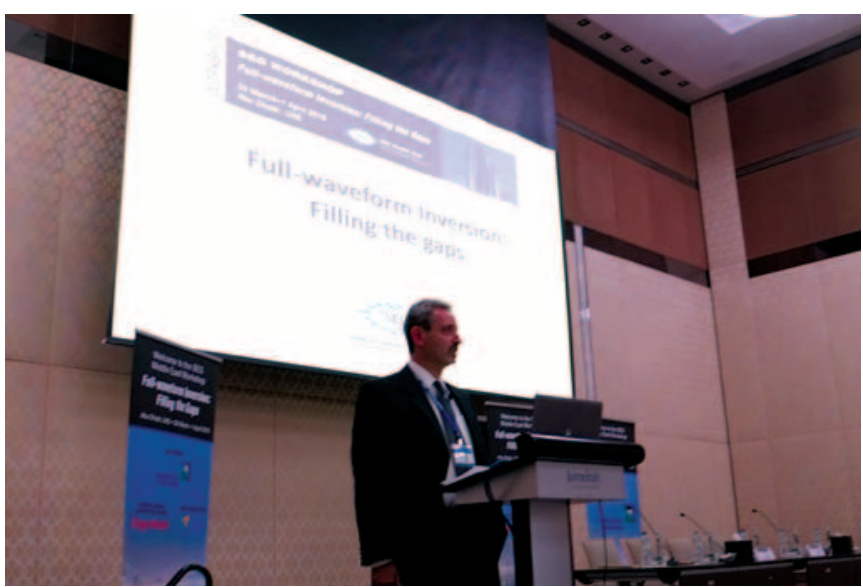

Wafik Beydoun (ADNOC) delivers the welcome speech for the SEG Middle East Workshop, "Full-waveform inversion: Filling the gaps." further research. During the workshops, participants witnessed and shared many advances, including more implementations on field data such as 3D and land. More companies are looking at the problem, including national companies, as they adapt this technology for their own purposes. Aramco, $\mathrm{ADNOC}, \mathrm{PDO}$, and many others are prime examples of this trend.

With extended discussion periods and time allotted for initializing and comparing ideas, the workshop proved hugely successful. There are still outstanding issues in FWI that require additional development and study of resolution, cost, application to inverting reservoir attributes or time-lapse monitoring, convergence to the appropriate model, the proper physics representation with elastic and anisotropic, viscoelastic assumptions, and many more. SEG looks forward to assessing the progress that will be made in the upcoming years and addressing the challenges that were identified and discussed at the workshop.

We extend our gratitude to all the presenters and participants for their informative speeches and discussions as well as sharing their knowledge and experience with the broader geoscience community. We are sincerely grateful to the organizing committee for diligently laying out the format and structure of the entire workshop. Last, the event would not have succeeded if not for the support and conviction of the SEG Middle East Office staff - Yogaani Bhatia, Ozlem Kocak, and Hyra Dalisay - through their logistical support and through relentlessly and patiently keeping us on track with the production schedule. TLE

\section{Organizing committee}

The organizing committee includes Wafik Beydoun (cochair), ADNOC; Tariq Alkhalifah (cochair), KAUST; Stephane Operto, CNRS Geosciences Azur; Thierry Tonellot, Saudi Aramco; Christoph Koeninger, Schlumberger; Aldo Vesnaver, Petroleum Institute; Anatoly Baumstein, ExxonMobil; Abdulla Al Kobaisi, ADNOC; Mamoru Takanashi, JOGMEC; Andrew Brenders, BP; Bertrand Duquet, Total; René-Édouard Plessix, Shell; and Ghiath Ajlani, CGG.

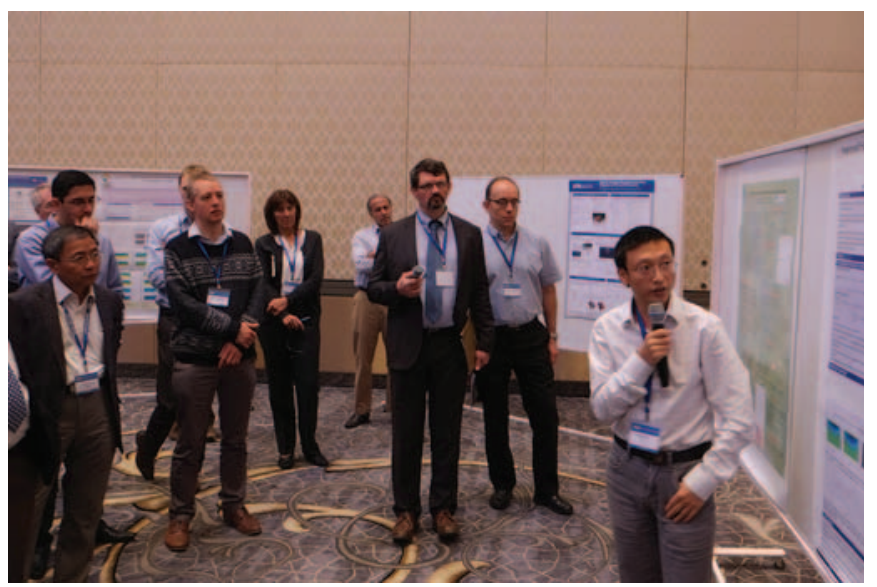

The FWI Workshop included two dedicated poster sessions with approximately 16 poster presentations. 